\title{
Associations between free sugars and nutrient intakes among children and adolescents in the UK
}

\author{
Sigrid Gibson $^{1 *}$, Lucy Francis ${ }^{1}$, Katie Newens ${ }^{2}$ and Barbara Livingstone ${ }^{3}$ \\ ${ }^{1}$ Sig-Nurture Ltd, Guildford, Surrey GU1 2TF, UK \\ ${ }^{2}$ Sugar Nutrition UK, London WC2R 1LA, UK \\ ${ }^{3}$ Ulster University, Belfast BT15 1ED, UK \\ (Submitted 12 February 2016 - Final revision received 20 July 2016 - Accepted 11 August 2016 - First published online 19 September 2016)
}

\section{Abstract}

This study explored associations between free sugars intake (using non-milk extrinsic sugars as proxy) and nutrient intakes among children aged 1.5-18 years in the UK National Diet and Nutrition Survey 2008-2012. Dietary records were completed by 2073 children (95\% completed 4 d). Mean free sugars intakes (\% energy) were $11 \cdot 8,14 \cdot 7$ and $15.4 \%$ in the $1 \cdot 5-3,4-10$ and $11-18$ years age groups, respectively. Nutrient intakes and nutrient density were compared across quintiles (Q1-Q5) of free sugars intake (\% energy) within each age group. Energy intake rose from Q1 to Q5 of free sugars, whereas percentages of energy intake from fat, SFA and protein dropped. Associations with micronutrients (mg/d or mcg/d) were mostly non-significant, but among 11-18-year-olds there were significant negative associations with $\mathrm{Zn}, \mathrm{Se}, \mathrm{Fe}, \mathrm{Cu}$, and vitamin $\mathrm{A}$ and $\mathrm{D}$. There were stronger negative associations with micronutrient density $(\mathrm{mg} / \mathrm{mcg}$ per $4 \cdot 18 \mathrm{MJ})$ for most nutrients in all age groups. Associations with vitamin $\mathrm{C}$ were positive. Results were similar after excluding misreporters. Children aged 4-18 years who consumed average amounts of free sugars or above ( $>13 \%$ energy or Q3-Q5) had lower diet quality than those consuming $<10 \%$ free sugars (Q1), but there were insufficient data to assess diets with $5 \%$ free sugars. High consumers obtained a higher proportion of free sugars from soft drinks, fruit juice and sugar confectionery and less from breakfast cereals. Ultimately, nutrient intakes depend on the total dietary pattern; however, reducing overconsumption of sugary foods and drinks with low nutrient density may help improve diet quality.

\section{Key words: Sugars: Diets: Micronutrient intakes: Recommendations}

Dietary guidelines around the world usually recommend that people reduce their 'sugar' intake, specifically referring to 'free sugars $^{,(1,2)}$ or added sugars ${ }^{(3)}$ intakes. These guidelines are mainly designed to reduce caries incidence ${ }^{(4,5)}$, although they are also intended to discourage overconsumption of energy ${ }^{(2,6)}$. Although all sugars are of equivalent energy density $(\mathrm{kJ} / \mathrm{g})$, terms such as intrinsic sugars and milk sugars, added sugars, free sugars and non-milk extrinsic sugars (NMES) are defined by source and nutritional considerations, rather than by chemical structure. Intrinsic sugars are not targeted for reduction because they exist within a cellular matrix with micronutrients and/or fibre (as in whole fruit), whereas milk sugars are less cariogenic, and milk itself is a key source of vital micronutrients such as Ca. In contrast, added sugars (sugars added during manufacture plus honey and syrups), free sugars (added sugars plus $100 \%$ fruit juice) and NMES (free sugars plus $50 \%$ of the sugar in processed and dried fruit) are specifically targeted for reduction on the premise that they are cariogenic, encourage passive overconsumption of energy and have the potential to compromise diet quality by displacing micronutrients from the diet. Intakes in the UK National Diet and Nutrition Survey (NDNS) are currently expressed as NMES, but for the purposes of this study the term 'free sugars' will be used based on the assumption that until new data become available ${ }^{(2)}$ they are sufficiently similar to NMES for assessment and monitoring purposes. The results based on NMES slightly overestimate free sugars intake, but we estimated this to be by less than $2 \mathrm{~g} / \mathrm{d}$ on average (see Methods section).

The micronutrient dilution hypothesis has some support in the literature, with several studies reporting inverse associations between dietary intakes of free/NMES/added sugars (\% energy) and micronutrient intakes ${ }^{(7-13)}$. Others have reported that the associations vary in both direction and size as well as by age group $^{(14)}$. We previously reported non-linear associations between dietary free sugars and micronutrient intakes, concluding that the negative impact on micronutrients was only seen at very high-sugar intake levels (e.g. > 20\% energy from NMES) because of displacement of nutrient-rich foods $\left.{ }^{(8,13}, 15\right)$.

On the other hand, diets very low in free sugars may also be unbalanced and low in nutrients. An analysis of data from the

Abbreviations: EAR, estimated average requirement; EI, energy intake; NDNS, National Diet and Nutrition Survey; NMES, non-milk extrinsic sugars.

* Corresponding author: S. Gibson, email sigrid@sig-nurture.com 
National Health and Nutrition Examination Survey (NHANES) III by the Institute of Medicine reported reduced intakes of $\mathrm{Ca}, \mathrm{Fe}$, $\mathrm{Zn}$ and vitamin $\mathrm{A}$ at low levels of added sugars ( $<5 \%$ energy) as well as at high levels (>25\% energy) ${ }^{(16)}$.

Free sugars are often consumed as constituents of, or as accompaniment to, nutrient-rich foods or drinks. They play an important part in making these foods palatable; examples include stewed fruit, breakfast cereals and yogurt. On the other hand, sugar-sweetened soft drinks provide energy and water but few micronutrients. Although their consumption is declining, they remain the major single source of free sugars for children, and especially teenagers, contributing to about one-third of intake among 11-18-year-olds. In the UK, Public Health England recommends that consumption of sugary soft drinks should be minimised for all ages ${ }^{(17)}$ and aims to reduce the intake of free sugars by restricting advertising and promotions, encouraging reformulation by manufacturers and adoption of healthy food policies in schools, hospitals and workplaces $^{(18)}$.

A comprehensive review of dietary surveys reported that total/free sugars intakes among children rose slightly between 1983 and 1997 and appear to have declined since ${ }^{(19)}$, especially among younger children. This may be because of heightened awareness among parents and caregivers of the potential health risks of overconsumption of free sugars and also greater availability of low-sugar/sugar-free beverages. On the other hand, free sugars intakes among older children and teenagers remain high. The focus of the present study was to explore current intakes of free sugars among children aged 18 months to 18 years participating in the NDNS Rolling Programme. Specifically, we evaluated the associations between quintiles of free sugars (NMES as proxy) in the diet and intakes of energy, macronutrients and micronutrients. As requirements are mostly expressed as absolute amounts and as diet quality is often expressed in terms of nutrient density, we have examined both.

\section{Methods}

National Diet and Nutrition Survey (Rolling Programme 2008-2012)

The NDNS is the most authoritative source of quantitative information on the food habits and nutrient intakes of the UK population. Jointly funded by the Department of Health in England (currently Public Health England) and the Food Standards Agency, the results are used by the Government to develop policy and monitor trends in diet and nutrient intakes. Households were sampled from the UK Postcode Address File, with either one adult plus one child (18 months or older) or one child being eligible for inclusion. Participants (or in the case of younger children, their carers) completed a detailed computerassisted personal interview to obtain background information and information on eating and lifestyle behaviours such as smoking, dieting, and medication and supplement use. Following the questionnaire, each survey participant (or their carer) was asked to complete a dietary record for 4 consecutive days, which provided detailed descriptions of each item consumed, time of consumption and amount, using household measures and photographs. As far as possible, the protocol was designed so that all days were equally represented over the whole sample. Repeat visits were made by trained interviewers to check records and probe for missing items. In the NDNS sample, 2008-2012, 55\% of the respondents completed at least $3 \mathrm{~d}$ of dietary assessment (the minimum for inclusion in the data set), and of these $95 \%$ completed $4 \mathrm{~d}$ of dietary assessments. Trained diet coders entered the food intake data from completed records using an in-house dietary assessment system at Medical Research Council Human Nutrition Research, DINO (Data In, Nutrients Out) ${ }^{(20)}$, using a databank of more than 7000 foods, which was regularly updated and extended for the survey. The compositional data included fortification nutrients. The food codes allowed the specification of items of different composition, in some cases down to brand level, more commonly at the sub-group level (e.g. "non-diet cola, own brand'). Volumes of tea, coffee and concentrated soft drinks included all water added as diluent. Milk was listed separately except for premixed tea and coffee from takeaway/vending sources. Similarly, table sugar signified discretionary sugar added to drinks or cereals. Data files reported food consumption and energy and nutrient intakes for each individual averaged over the number of days recorded. In addition, we extracted data on the sugar (NMES) content of each item to calculate the contribution of different sources to sugar intake.

\section{Non-milk extrinsic sugars as proxy for free sugars}

NMES include a notional $50 \%$ of the sugars present in dried fruit (these are not counted as free sugars). It was not possible to quantify the amount of dried fruit in composite products, but the mean intake of whole dried fruits (raisins, dates, prunes, mixed fruit) was $<2 \mathrm{~g} /$ person/d over the total population sample. Therefore, we estimated that the mean difference between NMES intake and free sugars intake was $<1 \mathrm{~g} / \mathrm{d}$, and for the purpose of the present analysis mean intakes of NMES and free sugars were assumed to be similar.

\section{Data analysis}

Data files from the core sample of 4156 participants from 4 years of the NDNS Rolling Programme (2008-2012) ${ }^{(21)}$ were obtained under licence from the UK Data Archive (http://www. esds.ac.uk). Our analysis included all children aged 18 years and under ( $n$ 2073). All data were weighted to adjust for sampling and non-response error. The distribution of sugars intakes (expressed as a percentage of total energy intake (EI)) was examined in each age group (1.5-3, 4-10, 11-18 years). Quintiles of percentage energy from sugars were calculated for each age group separately. Sexes were combined, as there was no significant difference in intakes (\% energy) between girls and boys in any age group.

Diet quality was assessed in terms of macronutrient and micronutrient intakes (excluding supplements) and also micronutrient density ( $\mathrm{mg}$ or $\mathrm{mcg} / 4184 \mathrm{~kJ}$ ( $1000 \mathrm{kcal})$ per $4 \cdot 18 \mathrm{MJ}$ ). Micronutrient adequacy (excluding supplements) was assessed by comparing the proportions of children with intakes 
below estimated average requirement (EAR and below lower reference nutrient intake $(\mathrm{LRNI})^{(5)}$.

All tests were two-sided using $P<0.05$ as criterion of significance. Differences between quintiles were assessed using ANOVA with tests for linear trends and additional planned contrasts between Q1 and other quintiles. Bonferroni's correction was used in post hoc multiple comparison tests. Misreporting (of energy) was assessed using the ratio of EI: BMR, with BMR calculated using the Henry equations ${ }^{(22)}$ as used by Scientific Advisory Committee on Nutrition in setting new Dietary Reference Values for Energy ${ }^{(23)}$. Subjects with missing values for body weight were assigned BMR values calculated from the mean values of other individuals of the same age and sex. We conducted sensitivity analysis to investigate whether results were valid after excluding children with EI outside $95 \%$ confidence limits of estimated energy requirements based on age group and sex. Age- and sex-specific values for physical activity level were obtained from the study by Black et al. and ranged from 1.57 (girls aged 1-6 years) to 1.75 (boys aged $12-17$ years).

Cut-off values for EI:BMR were calculated according to the method of Black et al. ${ }^{(24)}$ by applying values for $\mathrm{CV}_{\mathrm{wEI}}, \mathrm{CV}_{\mathrm{wB}}$ and $\mathrm{CV}_{\mathrm{tP}}$ of $23,8.5$ and 15 , respectively. The number of days of dietary recording $(\mathrm{d})$ was taken as 4

\section{Results}

\section{Sugars intake by age group (Table 1)}

Free sugars contributed on average 11.8 (SE 0.5 ) \% of energy among pre-school children ( $1.5-3$ years) ( $n$ 386), which is significantly less $(P<0.0001)$ compared with the $4-10$ years age group ( $n$ 803) (mean 14.7 (sE 0.3$) \%$ ) and the $11-18$ years age

Table 1. Mean intake of free sugars (\% energy) by age group (Mean values with their standard errors)

\begin{tabular}{|c|c|c|c|c|c|c|}
\hline & \multicolumn{5}{|c|}{ Free sugars (\% energy) (quintiles) } & \multirow[b]{2}{*}{ Total } \\
\hline & 1 & 2 & 3 & 4 & 5 & \\
\hline \multicolumn{7}{|l|}{$1.5-3$ years } \\
\hline$n$ & 83 & 76 & 77 & 76 & 74 & 386 \\
\hline Mean & $5 \cdot 3$ & 8.5 & 11.1 & $14 \cdot 3$ & $19 \cdot 4$ & 11.8 \\
\hline $\mathrm{SE}$ & 0.3 & 0.1 & 0.2 & 0.2 & 0.8 & 0.5 \\
\hline Median & 5.5 & 8.4 & 11.2 & 14.4 & $17 \cdot 7$ & $11 \cdot 2$ \\
\hline Minimum & 0.8 & $7 \cdot 1$ & 9.8 & $12 \cdot 6$ & $15 \cdot 7$ & 0.8 \\
\hline Maximum & $7 \cdot 1$ & 9.7 & $12 \cdot 6$ & $15 \cdot 7$ & 30.4 & 30.4 \\
\hline \multicolumn{7}{|l|}{$4-10$ years } \\
\hline$n$ & 174 & 149 & 160 & 163 & 157 & 803 \\
\hline Mean & $8 \cdot 1$ & 11.7 & 14.2 & $17 \cdot 1$ & 22.5 & 14.7 \\
\hline $\mathrm{SE}$ & 0.2 & 0.1 & 0.1 & 0.1 & 0.5 & 0.3 \\
\hline Median & 8.5 & 11.8 & $14 \cdot 2$ & $17 \cdot 1$ & 21.4 & 14.2 \\
\hline Minimum & 0.4 & $10 \cdot 3$ & $13 \cdot 0$ & $15 \cdot 4$ & $19 \cdot 0$ & 0.4 \\
\hline Maximum & $10 \cdot 3$ & 13.0 & $15 \cdot 4$ & $19 \cdot 0$ & 38.5 & 38.5 \\
\hline \multicolumn{7}{|l|}{$11-18$ years } \\
\hline$n$ & 181 & 172 & 165 & 183 & 183 & 884 \\
\hline Mean & 7.4 & 11.8 & 14.8 & $18 \cdot 2$ & $25 \cdot 1$ & $15 \cdot 4$ \\
\hline $\mathrm{SE}$ & 0.3 & 0.1 & 0.1 & 0.1 & 0.5 & 0.3 \\
\hline Median & 7.9 & 11.8 & 14.7 & $18 \cdot 1$ & $23 \cdot 8$ & 14.5 \\
\hline Minimum & 1.0 & $10 \cdot 2$ & $13 \cdot 2$ & 16.5 & 20.5 & 1.0 \\
\hline Maximum & $10 \cdot 2$ & $13 \cdot 2$ & $16 \cdot 4$ & 20.4 & 43.7 & $43 \cdot 7$ \\
\hline
\end{tabular}

group ( $n$ 884) (mean 15.4 (SE 0.3 ) \%). The two older groups were not significantly different from each other $(P=0.085)$. All quintiles contained a similar proportion of males and females, and there were no significant differences in ethnicity or socio-economic background (online Supplementary Table S1). Among all, $22 \%$ of all children had free sugars intakes supplying $<10 \%$ of energy (data not shown). The proportion was highest in pre-school children (42\%) compared with 18 and 19\% in the 4-10 years and 11-18 years age groups, respectively. Only $7 \%$ of pre-school children, $1 \%$ of $4-10$-year-olds and $4 \%$ of $11-18$-year-olds had intakes below $5 \%$ of energy. Among all children, $17 \%$ ( $7 \%$ of $<4$ years, $16 \%$ of $4-10$ years and $21 \%$ of $11-18$ years) had very high intakes of sugars ( $>20 \%$ energy).

\section{Analysis by quintiles of free sugars intake}

Free sugars intake was positively associated with EI in the two older groups $\left(P_{\text {trend }}<0.01\right)$ (Table 2$)$. There was a negative association with energy-adjusted protein, fat and SFA, and fibre intakes for all age groups. Absolute intakes of protein and fibre $(\mathrm{g} / \mathrm{d})$ also fell with increasing free sugars intake (online Supplementary Table S2). Among children aged $11-18$ years, there were inverse trends with intakes $(\mathrm{mg} / \mathrm{d}$ or $\mathrm{mcg} / \mathrm{d})$ of $\mathrm{Zn}$ and Se $(P<0.001)$, and weaker trends with vitamin A, vitamin $\mathrm{D}, \mathrm{Fe}$ and $\mathrm{Cu}(P<0.05)$ (online Supplementary Table S3). Intakes of other nutrients (thiamin, riboflavin, niacin, vitamin $\mathrm{B}_{6}$, vitamin $\mathrm{B}_{12}$, folate, vitamin $\mathrm{E}, \mathrm{Ca}, \mathrm{Mg}, \mathrm{K}, \mathrm{Na}, \mathrm{I})$ were not significantly different across quintiles. Among children under 11 years of age, there were no significant differences between quintiles apart from weak negative trends with vitamin $\mathrm{B}_{12}(P<0.05)$ and, in pre-school children, for I and vitamin $\mathrm{B}_{12}(P<0 \cdot 05)$. Free sugars intake was positively associated with vitamin $C$ intake in all age groups $(P<0.05)$.

When expressed as micronutrient density $(\mathrm{mg} / 4184 \mathrm{~kJ}$ $(1000 \mathrm{kcal})$ or per $4 \cdot 18 \mathrm{MJ})$, stronger negative trends with increasing free sugars intake were observed for most nutrients apart from vitamin C (Table 3). Differences between quintiles (planned contrasts) were most marked among 11-18-year-olds, with Q1 having significantly higher micronutrient density than Q3 or above, for six minerals (Fe, Mg, K, Cu, Zn, Se, I). Moreover, nutrient densities were higher in Q1 than in Q2 in the case of $\mathrm{Mg}, \mathrm{K}$ and $\mathrm{Zn}(P<0 \cdot 01)$. Among 4-10-year-olds, there was a significant difference between Q1 and Q2 for Se and I $(P<0.05)$. However, for the other fifteen nutrients in 11-18-year-olds and for sixteen nutrients in younger age groups, there was no significant difference in nutrient density between Q1 and Q2.

\section{Comparison with lower reference nutrient intake and estimated average requirement}

Overall, a few children aged under 11 years had intakes of micronutrients below the respective LRNI. However, among 11-18-year-olds, the prevalence was more than $10 \%$ for vitamin A, $\mathrm{Ca}, \mathrm{Fe}, \mathrm{Zn}, \mathrm{Mg}, \mathrm{K}, \mathrm{Se}$ and I (Table 4). There was no significant difference between quintiles. Prevalence estimates for intakes below EAR were correspondingly higher, but differences between 
Table 2. Energy and macronutrient intakes (\% energy) by quintiles of free sugars (\% energy), by age group (Mean values with their standard errors)

\begin{tabular}{|c|c|c|c|c|c|c|c|c|c|c|c|c|c|c|c|c|c|c|}
\hline & \multicolumn{6}{|c|}{$1.5-3$ years (free sugars (\% energy), quintiles) } & \multicolumn{6}{|c|}{$4-10$ years (free sugars (\% energy), quintiles) } & \multicolumn{6}{|c|}{$11-18$ years (free sugars (\% energy), quintiles) } \\
\hline & 1 & 2 & 3 & 4 & 5 & $P_{\text {trend }}$ & 1 & 2 & 3 & 4 & 5 & $P_{\text {trend }}$ & 1 & 2 & 3 & 4 & 5 & $P_{\text {trend }}$ \\
\hline$n$ & 83 & 76 & 77 & 76 & 74 & & 174 & 149 & 160 & 163 & 157 & & 181 & 172 & 165 & 183 & 183 & \\
\hline \multicolumn{19}{|c|}{ Total energy (kJ) } \\
\hline Mean & 4465 & 4798 & 4749 & 4757 & 5065 & 0.09 & 5871 & 6506 & 6498 & 6765 & 6682 & $<0.001$ & 6881 & 7440 & 7660 & 7842 & 7819 & 0.003 \\
\hline SE & 204 & 202 & 267 & 204 & 190 & & 164 & 184 & 149 & 169 & 165 & & 229 & 194 & 225 & 266 & 266 & \\
\hline \multicolumn{19}{|c|}{ Total energy (kcal) } \\
\hline Mean & 1059 & 1138 & 1125 & 1128 & 1200 & 0.09 & 1394 & 1544 & 1542 & 1605 & 1585 & $<0.001$ & 1635 & 1768 & 1820 & 1862 & 1855 & 0.003 \\
\hline $\mathrm{SE}$ & 48 & 48 & 63 & 49 & 45 & & 39 & 44 & 35 & 40 & 39 & & 55 & 46 & 54 & 63 & 63 & \\
\hline \multicolumn{19}{|c|}{ Percentage of energy from } \\
\hline \multicolumn{19}{|c|}{ Protein } \\
\hline Mean & $16 \cdot 9$ & $16 \cdot 5$ & $15 \cdot 1$ & 14.4 & 13.8 & $<0.001$ & $15 \cdot 6$ & 14.9 & 14.4 & $14 \cdot 0$ & $13 \cdot 1$ & $<0.001$ & $16 \cdot 8$ & $15 \cdot 8$ & $15 \cdot 0$ & 14.1 & 12.8 & $<0.001$ \\
\hline SE & 0.5 & 0.4 & 0.4 & 0.4 & 0.4 & & 0.3 & 0.2 & 0.3 & 0.2 & 0.3 & & 0.4 & 0.3 & 0.3 & 0.3 & 0.3 & \\
\hline \multicolumn{19}{|l|}{ Fat } \\
\hline Mean & $36 \cdot 6$ & 34.7 & 33.0 & 34.5 & 31.6 & 0.001 & 35.1 & $34 \cdot 3$ & 33.4 & 33.4 & 31.3 & $<0.001$ & 35.4 & 34.6 & 34.9 & 33.6 & 30.6 & $<0.001$ \\
\hline SE & 1.0 & 0.9 & 1.0 & 0.8 & 1.0 & & 0.7 & 0.5 & 0.6 & 0.5 & 0.5 & & 0.6 & 0.5 & 0.5 & 0.5 & 0.5 & \\
\hline \multicolumn{19}{|l|}{ SFA } \\
\hline Mean & $15 \cdot 8$ & $15 \cdot 2$ & 14.2 & $15 \cdot 0$ & $13 \cdot 4$ & 0.02 & 13.7 & $13 \cdot 3$ & 13.4 & $13 \cdot 2$ & $12 \cdot 4$ & 0.013 & 12.6 & $12 \cdot 7$ & 13.0 & 12.4 & 11.7 & 0.016 \\
\hline SE & 0.8 & 0.8 & 0.7 & 0.5 & 0.6 & & 0.4 & 0.3 & 0.3 & 0.3 & 0.3 & & 0.3 & 0.3 & 0.3 & 0.3 & 0.3 & \\
\hline \multicolumn{19}{|c|}{ Carbohydrate } \\
\hline Mean & 46.5 & $48 \cdot 8$ & 51.9 & 51.1 & 54.6 & $<0.001$ & $49 \cdot 3$ & $50 \cdot 8$ & 52.2 & $52 \cdot 7$ & $55 \cdot 6$ & $<0.001$ & 47.5 & 48.5 & $49 \cdot 6$ & 51.7 & 55.4 & $<0.001$ \\
\hline SE & 1.1 & 0.9 & 1.0 & 0.8 & 1.0 & & 0.7 & 0.5 & 0.6 & 0.5 & 0.6 & & 0.6 & 0.6 & 0.5 & 0.5 & 0.5 & \\
\hline \multicolumn{19}{|l|}{ Starch } \\
\hline Mean & $26 \cdot 4$ & $26 \cdot 2$ & $27 \cdot 3$ & 24.5 & 24.2 & 0.08 & $30 \cdot 8$ & $29 \cdot 8$ & 28.8 & $27 \cdot 1$ & $25 \cdot 4$ & $<0.001$ & 32.9 & 30.4 & 28.7 & 27.3 & 24.8 & $<0.001$ \\
\hline $\mathrm{SE}$ & 1.3 & $1 \cdot 1$ & 1.2 & 0.9 & 1.0 & & 0.7 & 0.5 & 0.6 & 0.5 & 0.5 & & 0.6 & 0.5 & 0.5 & 0.5 & 0.5 & \\
\hline \multicolumn{19}{|c|}{ Total sugars } \\
\hline Mean & $20 \cdot 1$ & $22 \cdot 6$ & 24.7 & 26.6 & 30.5 & $<0.001$ & 18.5 & $21 \cdot 0$ & $23 \cdot 4$ & $25 \cdot 6$ & $30 \cdot 3$ & $<0.001$ & 14.6 & $18 \cdot 2$ & 20.9 & 24.4 & 30.6 & $<0.001$ \\
\hline $\mathrm{SE}$ & $1 \cdot 1$ & 0.9 & 0.8 & 0.8 & 1.0 & & 0.6 & 0.4 & 0.4 & 0.4 & 0.5 & & 0.5 & 0.3 & 0.4 & 0.3 & 0.5 & \\
\hline \multicolumn{19}{|c|}{ Non-milk extrinsic sugars } \\
\hline Mean & 5.3 & 8.5 & $11 \cdot 1$ & 14.3 & $19 \cdot 4$ & $<0.001$ & $8 \cdot 1$ & $11 \cdot 7$ & $14 \cdot 2$ & $17 \cdot 1$ & 22.5 & $<0.001$ & $7 \cdot 4$ & 11.8 & $14 \cdot 8$ & $18 \cdot 2$ & $25 \cdot 1$ & $<0.001$ \\
\hline SE & 0.3 & 0.1 & 0.2 & 0.2 & 0.8 & & 0.2 & 0.1 & 0.1 & 0.1 & 0.5 & & 0.3 & 0.1 & 0.1 & 0.1 & 0.5 & \\
\hline \multicolumn{19}{|c|}{ NSP fibre density (g/4184 kJ (1000 kcal)) } \\
\hline Mean & 7.8 & $7 \cdot 8$ & 7.2 & 7.0 & 6.7 & 0.014 & $7 \cdot 8$ & $7 \cdot 7$ & 7.6 & $7 \cdot 1$ & 6.5 & $<0.001$ & 7.8 & 7.8 & $7 \cdot 2$ & 7.0 & $6 \cdot 7$ & $<0.001$ \\
\hline SE & 0.5 & 0.4 & 0.4 & 0.4 & 0.3 & & 0.3 & 0.2 & 0.2 & 0.2 & 0.2 & & 0.5 & 0.4 & 0.4 & 0.4 & 0.3 & \\
\hline
\end{tabular}


Table 3. Micronutrient density (ug or $\mathrm{mg} / 4.18 \mathrm{MJ}$ ) by quintiles of free sugars (\% energy), by age group (Mean values with their standard errors)

\begin{tabular}{|c|c|c|c|c|c|c|c|c|c|c|c|c|c|c|c|c|c|c|}
\hline & \multicolumn{6}{|c|}{$1.5-3$ years (free sugars (\% energy), quintiles) } & \multicolumn{6}{|c|}{$4-10$ years (free sugars (\% energy), quintiles) } & \multicolumn{6}{|c|}{$11-18$ years (energy from free sugars, quintiles) } \\
\hline & 1 & 2 & 3 & 4 & 5 & $P_{\text {trend }}$ & 1 & 2 & 3 & 4 & 5 & $P_{\text {trend }}$ & 1 & 2 & 3 & 4 & 5 & $P_{\text {trend }}$ \\
\hline$n$ & 83 & 76 & 77 & 76 & 74 & & 174 & 149 & 160 & 163 & 157 & & 181 & 172 & 165 & 183 & 183 & \\
\hline \multicolumn{19}{|c|}{ Vitamin A (retinol equivalents) } \\
\hline Mean & 568.0 & $472 \cdot 6$ & 489.3 & 458.9 & $375 \cdot 1$ & 0.039 & 463.0 & $455 \cdot 8$ & 456.9 & $416 \cdot 3$ & 356.9 & 0.029 & $453.7^{b}$ & 423.7 & 343.7 & 330.5 & $324 \cdot 3^{\mathrm{a}}$ & 0.001 \\
\hline $\mathrm{SE}$ & 72.4 & $49 \cdot 3$ & 59.9 & 78.0 & 35.9 & & 38.3 & 44.5 & 41.0 & $28 \cdot 8$ & $23 \cdot 0$ & & $48 \cdot 3$ & 31.0 & $26 \cdot 9$ & $25 \cdot 2$ & $22 \cdot 7$ & \\
\hline \multicolumn{19}{|l|}{ Thiamin } \\
\hline Mean & 0.9 & 0.9 & 0.9 & 0.8 & 0.8 & 0.023 & 0.8 & 0.9 & 0.9 & 0.8 & 0.8 & 0.048 & 0.9 & 0.8 & 0.8 & 0.8 & 0.7 & $<0.001$ \\
\hline SE & 0.0 & 0.0 & 0.0 & 0.0 & 0.0 & & 0.0 & 0.0 & 0.0 & 0.0 & 0.0 & & 0.0 & 0.0 & 0.0 & 0.0 & 0.0 & \\
\hline \multicolumn{19}{|c|}{ Riboflavin } \\
\hline Mean & 1.4 & 1.4 & 1.3 & 1.2 & $1 \cdot 1$ & 0.002 & $1.0^{\mathrm{b}}$ & $1.0^{\mathrm{b}}$ & $1.0^{\mathrm{b}}$ & 0.9 & $0.9^{\mathrm{a}}$ & $<0.001$ & 0.9 & 0.8 & 0.8 & 0.8 & 0.8 & 0.074 \\
\hline $\mathrm{SE}$ & 0.1 & 0.1 & 0.1 & 0.1 & 0.1 & & 0.0 & 0.0 & 0.0 & 0.0 & 0.0 & & 0.0 & 0.0 & 0.0 & 0.0 & 0.0 & \\
\hline \multicolumn{19}{|c|}{ Niacin equivalents } \\
\hline Mean & $19 \cdot 1^{\mathrm{b}}$ & 18.4 & $16 \cdot 9$ & $15 \cdot 6^{\mathrm{a}}$ & $15 \cdot 8^{\mathrm{a}}$ & $<0.001$ & $18 \cdot 0^{\mathrm{b}}$ & $18.0^{\mathrm{b}}$ & $17 \cdot 3$ & $16 \cdot 8$ & $15 \cdot 7^{\mathrm{a}}$ & $<0.001$ & $20.5^{\mathrm{b}}$ & $19 \cdot 6$ & $18 \cdot 2$ & $17 \cdot 5^{\mathrm{a}}$ & $17 \cdot 3^{\mathrm{a}}$ & $<0.001$ \\
\hline $\mathrm{SE}$ & 1.1 & 0.7 & 0.7 & 0.6 & 0.5 & & 0.5 & 0.6 & 0.5 & 0.5 & 0.4 & & 0.7 & 0.5 & 0.5 & 0.5 & 0.9 & \\
\hline \multicolumn{19}{|c|}{ Vitamin $B_{6}$} \\
\hline Mean & 1.4 & 1.3 & 1.2 & $1 \cdot 1$ & $1 \cdot 1$ & 0.004 & $1 \cdot 2$ & 1.2 & 1.2 & $1 \cdot 1$ & 1.0 & 0.004 & 1.2 & $1 \cdot 2$ & $1 \cdot 1$ & $1 \cdot 1$ & 1.3 & 0.592 \\
\hline SE & 0.1 & 0.1 & 0.1 & 0.0 & 0.1 & & 0.0 & 0.0 & 0.1 & 0.0 & 0.0 & & 0.0 & 0.1 & 0.1 & 0.1 & 0.1 & \\
\hline \multicolumn{19}{|c|}{ Vitamin $B_{12}$} \\
\hline Mean & $4 \cdot 1^{b}$ & 3.6 & 3.6 & $3 \cdot 1$ & $2 \cdot 8^{\mathrm{a}}$ & 0.000 & $2 \cdot 9^{b}$ & $2 \cdot 6$ & $2 \cdot 5$ & $2 \cdot 5^{\mathrm{a}}$ & $2 \cdot 2^{\mathrm{a}}$ & $<0.001$ & $2 \cdot 6^{b}$ & $2 \cdot 3$ & $2 \cdot 3$ & $2 \cdot 1^{\mathrm{a}}$ & $2 \cdot 2$ & 0.006 \\
\hline $\mathrm{SE}$ & 0.3 & 0.3 & 0.3 & 0.2 & 0.2 & & 0.1 & $0 \cdot 1$ & 0.1 & $0 \cdot 1$ & 0.1 & & 0.1 & 0.1 & 0.1 & 0.1 & 0.1 & \\
\hline \multicolumn{19}{|l|}{ Folate } \\
\hline Mean & $140 \cdot 2$ & $143 \cdot 2$ & $142 \cdot 2$ & 124.4 & $128 \cdot 3$ & 0.079 & 135.5 & 128.9 & $135 \cdot 4$ & $125 \cdot 8$ & 118.7 & 0.009 & $132 \cdot 1^{b}$ & $127 \cdot 1^{b}$ & 114.6 & 117.6 & $106 \cdot 6^{\mathrm{a}}$ & $<0.001$ \\
\hline $\mathrm{SE}$ & 6.6 & $6 \cdot 8$ & $9 \cdot 1$ & 5.9 & 8.9 & & 4.9 & 3.9 & 4.7 & 4.6 & 3.7 & & $4 \cdot 8$ & 4.6 & 4.4 & 4.6 & 4.0 & \\
\hline \multicolumn{19}{|c|}{ Vitamin C } \\
\hline Mean & $55 \cdot 0$ & 54.9 & $55 \cdot 9$ & $64 \cdot 2$ & $70 \cdot 0$ & 0.077 & $45 \cdot 5^{\mathrm{a}}$ & $50 \cdot 3^{a}$ & 57.4 & $59 \cdot 2$ & $69.8^{\mathrm{b}}$ & $<0.001$ & $39.5^{\mathrm{a}}$ & $39.0^{\mathrm{a}}$ & 47.5 & 49.4 & $55 \cdot 1^{\mathrm{b}}$ & $<0.001$ \\
\hline $\mathrm{SE}$ & 7.6 & $5 \cdot 7$ & 6.5 & 8.0 & 6.9 & & 2.9 & 3.7 & 3.7 & 3.6 & 4.3 & & 3.1 & $2 \cdot 6$ & 3.6 & 3.7 & $5 \cdot 1$ & \\
\hline \multicolumn{19}{|c|}{ Vitamin D } \\
\hline Mean & $2 \cdot 2$ & 1.7 & 1.4 & 1.7 & 1.5 & 0.230 & 1.5 & 1.4 & 1.2 & 1.2 & 1.2 & 0.009 & $1.4^{b}$ & 1.3 & 1.2 & 1.2 & $1.0^{\mathrm{a}}$ & $<0.001$ \\
\hline $\mathrm{SE}$ & 0.5 & 0.4 & 0.2 & 0.3 & 0.2 & & 0.1 & 0.1 & 0.1 & 0.1 & 0.1 & & 0.1 & 0.1 & 0.1 & 0.1 & 0.1 & \\
\hline \multicolumn{19}{|c|}{ Vitamin $\mathrm{E}$} \\
\hline Mean & 4.9 & 4.4 & 4.5 & $4 \cdot 3$ & $4 \cdot 2$ & 0.092 & 4.9 & 4.9 & 4.6 & 4.6 & $4 \cdot 3$ & 0.004 & $5 \cdot 2^{b}$ & $4.9^{b}$ & 4.7 & 4.7 & $4 \cdot 2^{\mathrm{a}}$ & $<0.001$ \\
\hline SE & 0.4 & 0.3 & 0.3 & 0.3 & 0.2 & & 0.2 & 0.2 & 0.2 & $0 \cdot 1$ & 0.2 & & 0.2 & 0.2 & 0.1 & 0.2 & 0.1 & \\
\hline \multicolumn{19}{|l|}{$\mathrm{Fe}$} \\
\hline Mean & $5 \cdot 7$ & $6 \cdot 0$ & 5.5 & 5.5 & 5.5 & 0.399 & $5 \cdot 8$ & $5 \cdot 9^{a}$ & $5 \cdot 8$ & $5 \cdot 6$ & $5 \cdot 3^{b}$ & 0.006 & $6 \cdot 1^{b}$ & $5 \cdot 7$ & $5 \cdot 4^{\mathrm{a}}$ & $5 \cdot 2^{a}$ & $4 \cdot 8^{\mathrm{a}}$ & $<0.001$ \\
\hline SE & 0.3 & 0.4 & 0.3 & 0.3 & 0.3 & & 0.2 & 0.2 & 0.2 & 0.2 & 0.1 & & 0.2 & 0.1 & 0.1 & 0.1 & 0.1 & \\
\hline \multicolumn{19}{|l|}{$\mathrm{Ca}$} \\
\hline Mean & $750 \cdot 4$ & 733.5 & $702 \cdot 8$ & $646 \cdot 9$ & $607 \cdot 7$ & 0.001 & $559 \cdot 6^{\mathrm{b}}$ & $548 \cdot 6^{\mathrm{b}}$ & 533.6 & $507 \cdot 3$ & $463 \cdot 7^{\mathrm{a}}$ & 0.000 & 464.8 & 452.6 & 437.2 & 426.7 & 417.4 & 0.007 \\
\hline SE & 45.9 & $28 \cdot 8$ & $37 \cdot 6$ & 33.7 & $30 \cdot 2$ & & $20 \cdot 7$ & $21 \cdot 1$ & $15 \cdot 3$ & $15 \cdot 1$ & 14.2 & & $15 \cdot 6$ & 13.5 & 13.7 & $13 \cdot 3$ & $14 \cdot 1$ & \\
\hline \multicolumn{19}{|l|}{$\mathrm{Mg}$} \\
\hline Mean & $143 \cdot 1$ & 144.2 & 136.7 & $130 \cdot 6$ & 128.8 & 0.001 & $130 \cdot 1^{b}$ & $126 \cdot 2$ & $130 \cdot 1^{b}$ & 123.5 & $119 \cdot 3^{\mathrm{a}}$ & 0.003 & $129 \cdot 5^{\mathrm{b}}$ & 121.3 & $116 \cdot 0^{\mathrm{a}}$ & $113 \cdot 8^{a}$ & $108 \cdot 2^{\mathrm{a}}$ & $<0.001$ \\
\hline SE & 4.8 & 3.4 & 4.1 & 3.7 & 4.0 & & $2 \cdot 7$ & $2 \cdot 4$ & $2 \cdot 9$ & $2 \cdot 3$ & 2.4 & & 3.1 & $2 \cdot 1$ & $2 \cdot 2$ & $2 \cdot 1$ & $2 \cdot 1$ & \\
\hline $\mathrm{K}$ & & & & & & & & & & & & & & & & & & \\
\hline Mean & $1700 \cdot 8^{b}$ & $1679 \cdot 0$ & $1609 \cdot 9$ & $1532 \cdot 2$ & $1480 \cdot 7^{a}$ & $<0.001$ & $1477 \cdot 5^{\mathrm{b}}$ & $1420 \cdot 4$ & 1431.8 & 1393.8 & $1351 \cdot 4^{\mathrm{a}}$ & 0.004 & $1431 \cdot 9^{b}$ & $1341 \cdot 2$ & $1308 \cdot 6^{a}$ & $1292 \cdot 0^{\mathrm{a}}$ & $1192 \cdot 9^{a}$ & $<0.001$ \\
\hline SE & $51 \cdot 3$ & 38.6 & $45 \cdot 4$ & $52 \cdot 6$ & $59 \cdot 0$ & & $32 \cdot 1$ & 31.4 & $30 \cdot 3$ & $30 \cdot 1$ & $28 \cdot 9$ & & $36 \cdot 7$ & $25 \cdot 0$ & $28 \cdot 4$ & 28.9 & $30 \cdot 0$ & \\
\hline $\mathrm{Zn}$ & & & & & & & & & & & & & & & & & & \\
\hline Mean & $5 \cdot 2^{b}$ & 4.9 & 4.5 & $4.4^{\mathrm{a}}$ & $4 \cdot 2^{\mathrm{a}}$ & $<0.001$ & 4.5 & 4.4 & 4.1 & 4.0 & 3.7 & $<0.001$ & $4.9^{b}$ & $4.4^{\mathrm{a}}$ & $4 \cdot 2^{\mathrm{a}}$ & $3.9^{\mathrm{a}}$ & $3.6^{\mathrm{a}}$ & $<0.001$ \\
\hline $\mathrm{SE}$ & 0.2 & 0.2 & 0.1 & 0.2 & 0.2 & & 0.2 & 0.1 & 0.1 & $0 \cdot 1$ & 0.1 & & 0.1 & 0.1 & 0.1 & 0.1 & 0.1 & \\
\hline
\end{tabular}


quintiles mostly failed to reach statistical significance, except for Zn among 11-18-year-olds, where the risk of intake below EAR was significantly higher for Q5 compared with Q1, Q2 and Q3 $(P<0 \cdot 05)$.

\section{Misreporting}

Approximately $27 \%$ of children were classified as suspected under-reporters of energy, whereas only $0.3 \%$ were overreporters. Exclusion of misreporters resulted in higher mean energy and micronutrient intakes $(\mathrm{mg} / \mathrm{d}$ or $\mathrm{mcg} / \mathrm{d}$ ), especially in Q1, and weaker associations between free sugars intake and energy (data not shown). However, findings for nutrient density were similar to the total sample, with significant inverse trends for most nutrients in the two older groups.

\section{Sources of free sugars}

Soft drinks were the most important single contributor to free sugars intakes, supplying 17,18 and $33 \%$ of free sugars among age groups 1.5-3, 4-10 and 11-18 years, respectively (Table 5). High consumers of free sugars consumed a higher proportion of their free sugars in the form of sugar-sweetened soft drinks, compared with low consumers $\left(P_{\text {trend }}<0 \cdot 001\right)$; there were also significant positive trends for fruit juice and sugar confectionery $\left(P_{\text {trend }}<0 \cdot 001\right)$. Conversely, breakfast cereals and milk and yogurt provided a lower proportion of free sugars among high consumers $\left(P_{\text {trend }}<0 \cdot 001\right)$.

\section{Discussion}

The findings of the present study broadly concur with other recent studies ${ }^{(25-27)}$. Overall, the level of free sugars (as a percentage of total dietary energy) was inversely associated with diet quality, as measured by nutrient density. Associations with vitamin $\mathrm{C}$ were positive, due to the contribution from fruit juice. Significant micronutrient dilution was observed at Q3 (13\%) and above, suggesting that the majority of children may benefit from free sugars reduction, provided this was from food and beverages low in micronutrients. There was less evidence that below-average consumers (Q2 and below) would benefit in terms of micronutrient intakes, and insufficient evidence to assess very low free sugars intakes (about 5\% energy) due to low numbers.

In a recent study in Australian children and adolescents, Louie \& Tapsell ${ }^{(26)}$ reported that small but consistent negative associations were found between added sugars (\% energy) and most nutrients, whereas associations with total sugars (\% energy) were inconsistent and weaker. The UK (and WHO) definition of free sugars falls somewhere between added sugars and total sugars, by including fruit juice and purées yet excluding sugars in whole fruit and milk, which exert a positive influence on diet quality. In reviewing the evidence from twenty-two studies, Louie \& Tapsell ${ }^{(27)}$ commented that the magnitude of the negative effect of added sugars was generally small and may not be of clinical significance. In Irish children and adolescents, Joyce \& Gibney $^{(28)}$ reported a general 
Table 4. Prevalence of intakes below lower reference nutrient intake (LRNI) and estimated average requirement (EAR)

\begin{tabular}{|c|c|c|c|c|c|c|c|c|c|c|c|c|c|c|c|}
\hline & \multicolumn{5}{|c|}{$1.5-3$ years (free sugars (\% energy), quintiles) } & \multicolumn{5}{|c|}{$4-10$ years (free sugars (\% energy), quintiles) } & \multicolumn{5}{|c|}{$11-18$ years (free sugars (\% energy), quintiles) } \\
\hline & 1 & 2 & 3 & 4 & 5 & 1 & 2 & 3 & 4 & 5 & 1 & 2 & 3 & 4 & 5 \\
\hline$n$ & 83 & 76 & 77 & 76 & 74 & 143 & 131 & 139 & 150 & 143 & 74 & 93 & 87 & 112 & 114 \\
\hline \multicolumn{16}{|l|}{ Below LRNI* } \\
\hline Vitamin A (\%) & 4.6 & 9.7 & 9.6 & 6.7 & $5 \cdot 7$ & 6.9 & 5.8 & $5 \cdot 3$ & 4.3 & 8.5 & 12.5 & 9.9 & $12 \cdot 1$ & 15.7 & $16 \cdot 0$ \\
\hline Thiamin (\%) & 0.0 & 0.0 & 0.0 & 0.0 & 0.0 & 0.0 & 0.0 & 0.0 & 0.0 & 0.0 & 0.0 & 0.0 & 0.0 & 0.0 & 0.0 \\
\hline Riboflavin (\%) & 0.0 & 0.0 & 2.9 & 0.0 & 0.0 & 1.0 & 0.0 & 0.7 & 0.4 & $2 \cdot 2$ & 18.4 & $14 \cdot 3$ & 13.5 & $14 \cdot 2$ & 13.6 \\
\hline Vitamin $\mathrm{B}_{6}(\%)$ & 0.0 & 0.0 & 0.0 & 0.0 & 0.0 & 0.0 & 0.0 & 0.0 & 0.0 & 0.0 & 0.0 & 0.0 & 0.0 & 0.0 & 0.5 \\
\hline Vitamin $\mathrm{B}_{12}(\%)$ & 0.0 & 0.0 & 0.0 & 0.0 & 0.0 & 0.3 & 0.4 & 0.0 & 0.0 & 0.0 & 3.1 & 1.6 & 1.2 & 0.3 & $2 \cdot 2$ \\
\hline Folate (\%) & 0.0 & 0.0 & 2.9 & 0.0 & 0.0 & 0.0 & 0.0 & 0.0 & 0.0 & 0.0 & 4.3 & $2 \cdot 7$ & $6 \cdot 3$ & 5.8 & $10 \cdot 0$ \\
\hline Vitamin C (\%) & 0.0 & 0.0 & $2 \cdot 9$ & 0.0 & 0.0 & 0.7 & 0.0 & 0.0 & 0.0 & 0.0 & 1.7 & 0.0 & $2 \cdot 0$ & 0.0 & 1.8 \\
\hline $\mathrm{Fe}(\%)$ & $13 \cdot 6$ & $2 \cdot 7$ & $7 \cdot 6$ & 6.9 & 4.3 & 3.5 & $2 \cdot 4$ & $1 \cdot 1$ & 0.4 & 0.0 & $27 \cdot 9$ & $20 \cdot 3$ & $24 \cdot 8$ & 25.5 & 32.7 \\
\hline $\mathrm{Ca}(\%)$ & 0.0 & 0.0 & $2 \cdot 9$ & 0.0 & 0.0 & 4.6 & $2 \cdot 2$ & 0.6 & 0.0 & $2 \cdot 6$ & $18 \cdot 0$ & 8.6 & $12 \cdot 0$ & 13.8 & $14 \cdot 1$ \\
\hline $\mathrm{Mg}(\%)$ & 0.0 & 0.0 & 2.9 & 0.0 & 0.0 & $2 \cdot 9$ & 2.5 & 1.0 & 0.8 & 1.7 & 40.9 & $36 \cdot 4$ & $36 \cdot 4$ & 35.2 & 47.5 \\
\hline $\mathrm{K}(\%)$ & 0.0 & 0.0 & 2.9 & 0.0 & 0.0 & 0.7 & 0.0 & 0.0 & 0.0 & 0.0 & 24.4 & 21.5 & $22 \cdot 1$ & $22 \cdot 2$ & 28.2 \\
\hline $\mathrm{Zn}(\%)$ & 6.7 & 0.0 & 5.0 & 8.7 & $5 \cdot 8$ & 8.5 & 7.1 & 4.6 & 7.4 & 15.9 & 12.5 & $11 \cdot 2$ & $12 \cdot 0$ & $16 \cdot 4$ & 26.9 \\
\hline Se (\%) & 0.0 & 0.0 & 2.9 & 0.0 & 0.0 & $2 \cdot 0$ & 0.0 & 0.3 & 0.4 & 2.9 & 31.9 & 29.5 & 29.2 & 26.6 & 45.6 \\
\hline I (\%) & 3.7 & 0.0 & 2.9 & 0.0 & 0.0 & 4.8 & 1.9 & $2 \cdot 6$ & $2 \cdot 6$ & 6.6 & $15 \cdot 8$ & 11.7 & 14.9 & 14.4 & $18 \cdot 4$ \\
\hline \multicolumn{16}{|l|}{ Below EAR $\dagger$} \\
\hline Vitamin A (\%) & 14.4 & $20 \cdot 8$ & $22 \cdot 7$ & $22 \cdot 5$ & $18 \cdot 6$ & 22.5 & $17 \cdot 6$ & 17.5 & $16 \cdot 8$ & 23.9 & $32 \cdot 7$ & $25 \cdot 1$ & $37 \cdot 0$ & 39.2 & 39.7 \\
\hline Thiamin (\%) & 6.6 & 0.7 & $2 \cdot 9$ & 0.0 & $2 \cdot 4$ & 6.5 & 1.0 & 3.6 & $2 \cdot 3$ & $5 \cdot 0$ & $15 \cdot 6$ & $16 \cdot 0$ & $14 \cdot 1$ & $16 \cdot 7$ & $24 \cdot 2$ \\
\hline Riboflavin (\%) & $3 \cdot 7$ & 0.0 & $2 \cdot 9$ & 4.0 & 0.0 & 6.9 & 6.6 & 3.4 & $4 \cdot 1$ & 8.2 & $26 \cdot 5$ & $21 \cdot 0$ & $21 \cdot 6$ & $24 \cdot 3$ & $22 \cdot 3$ \\
\hline Vitamin $\mathrm{B}_{6}(\%)$ & 0.0 & 0.0 & 0.0 & 0.0 & 0.0 & 0.0 & 0.0 & 0.0 & 0.0 & 0.0 & 0.0 & 1.3 & 0.0 & 0.0 & 0.5 \\
\hline Vitamin $\mathrm{B}_{12}(\%)$ & 0.0 & 0.0 & 2.9 & 0.0 & 0.0 & 0.3 & 0.4 & 0.0 & 0.0 & 0.0 & 3.8 & $2 \cdot 9$ & 1.2 & 1.3 & 4.2 \\
\hline Folate (\%) & 0.0 & 0.0 & $2 \cdot 9$ & 0.0 & 0.0 & $3 \cdot 7$ & 0.5 & 0.7 & $2 \cdot 9$ & 4.5 & 24.4 & 17.9 & $20 \cdot 3$ & $23 \cdot 2$ & 31.3 \\
\hline Vitamin C (\%) & $6 \cdot 2$ & 3.3 & 5.9 & $2 \cdot 8$ & 1.9 & $5 \cdot 1$ & $2 \cdot 3$ & $2 \cdot 1$ & $1 \cdot 1$ & 0.4 & $9 \cdot 1$ & 9.4 & $10 \cdot 5$ & $4 \cdot 3$ & $4 \cdot 1$ \\
\hline $\mathrm{Fe}(\%)$ & 43.4 & $26 \cdot 9$ & $32 \cdot 2$ & $35 \cdot 3$ & 24.4 & $12 \cdot 0$ & $10 \cdot 9$ & $7 \cdot 3$ & 4.9 & $12 \cdot 2$ & $60 \cdot 0$ & $53 \cdot 8$ & $51 \cdot 3$ & 59.2 & 60.8 \\
\hline $\mathrm{Ca}(\%)$ & 3.7 & 0.7 & $2 \cdot 9$ & 0.0 & 0.0 & 6.5 & $7 \cdot 7$ & $2 \cdot 3$ & 1.2 & 8.4 & $47 \cdot 7$ & 43.0 & 35.9 & $47 \cdot 3$ & $47 \cdot 1$ \\
\hline $\mathrm{Mg}(\%)$ & 0.0 & $0 . .0$ & 2.9 & 0.0 & 0.0 & $10 \cdot 1$ & 8.6 & 6.6 & 6.9 & $12 \cdot 1$ & 72.4 & 70.9 & 74.2 & 69.5 & 77.7 \\
\hline $\mathrm{K}(\%)$ & 0.0 & 0.0 & 2.9 & 0.0 & 0.0 & 4.7 & 3.4 & 3.5 & $2 \cdot 7$ & 5.1 & $66 \cdot 7$ & 62.9 & 61.2 & 58.1 & 71.4 \\
\hline $\mathrm{Zn}(\%)$ & $12 \cdot 7$ & $12 \cdot 6$ & 21.3 & $20 \cdot 8$ & 18.6 & 30.4 & 19.9 & $18 \cdot 7$ & 24.0 & 35.6 & $35 \cdot 6^{\mathrm{a}}$ & $35.0^{\mathrm{a}}$ & $37.2^{\mathrm{a}}$ & 56.6 & $59.6^{b}$ \\
\hline $\mathrm{Se}(\%)$ & 3.0 & 1.0 & 4.6 & 4.6 & 0.9 & 4.7 & 8.5 & 3.5 & 3.9 & 9.3 & 53.8 & 50.2 & 50.1 & 56.1 & 67.5 \\
\hline I (\%) & 6.4 & 0.9 & 4.6 & 11.8 & 3.0 & 14.0 & 22.0 & $12 \cdot 4$ & 12.5 & 21.0 & 50.4 & 37.6 & $42 \cdot 7$ & 46.4 & 47.4 \\
\hline
\end{tabular}

a,b Mean values with unlike superscript letters were significantly different.

* Prevalence of intakes below LRNI was not significantly different between quintiles.

*Prevalence of intakes below LRNI was not significantly different between quintiles.
† Prevalence of intakes below EAR was only significant for $Z n$ in the $11-18$ years age group. 
Table 5. Sources of free sugars by quintile

(Mean values with their standard errors)

\begin{tabular}{|c|c|c|c|c|c|c|c|c|c|c|c|c|c|c|c|c|c|c|}
\hline & \multicolumn{6}{|c|}{$1.5-3$ years (free sugars (\% energy), quintiles) } & \multicolumn{6}{|c|}{ 4-10 years (free sugars (\% energy), quintiles) } & \multicolumn{6}{|c|}{$11-18$ years (free sugars (\% energy), quintiles) } \\
\hline & 1 & 2 & 3 & 4 & 5 & $P_{\text {trend }}$ & 1 & 2 & 3 & 4 & 5 & $P_{\text {trend }}$ & 1 & 2 & 3 & 4 & 5 & $P_{\text {trend }}$ \\
\hline$n$ & 83 & 76 & 77 & 76 & 74 & & 174 & 149 & 160 & 163 & 157 & & 181 & 172 & 165 & 183 & 183 & \\
\hline \multicolumn{19}{|c|}{ Free sugars $(g / d)$} \\
\hline Mean & $15 \cdot 1$ & $25 \cdot 7$ & 33.4 & 43.0 & $62 \cdot 2$ & & 30.1 & $48 \cdot 2$ & $58 \cdot 3$ & $73 \cdot 1$ & 95.0 & & $32 \cdot 8$ & $55 \cdot 8$ & 71.7 & $90 \cdot 4$ & $123 \cdot 2$ & \\
\hline SE & 1.1 & $1 \cdot 2$ & 1.9 & 1.8 & $3 \cdot 3$ & & $1 \cdot 2$ & 1.4 & 1.4 & $2 \cdot 0$ & $3 \cdot 1$ & & $1 \cdot 6$ & $1 \cdot 6$ & $2 \cdot 2$ & 3.1 & 4.6 & \\
\hline \multirow{2}{*}{\multicolumn{19}{|c|}{$\begin{array}{l}\text { Percentage of free sugars from selected sources } \\
\text { Milk and yogurt }\end{array}$}} \\
\hline & & & & & & & & & & & & & & & & & & \\
\hline Mean & $20 \cdot 8$ & $16 \cdot 5$ & 13.5 & $12 \cdot 3$ & 8.6 & \multirow[t]{2}{*}{$<0.01$} & $9 \cdot 8$ & 7.7 & 9.0 & $6 \cdot 2$ & 5.4 & \multirow[t]{2}{*}{$<0.01$} & $5 \cdot 6$ & $5 \cdot 0$ & $4 \cdot 1$ & 3.2 & 4.5 & \multirow[t]{2}{*}{ NS } \\
\hline SE & 4.5 & $2 \cdot 7$ & $2 \cdot 7$ & $2 \cdot 1$ & $1 \cdot 8$ & & 1.5 & $1 \cdot 2$ & $1 \cdot 1$ & 0.9 & 0.8 & & $1 \cdot 2$ & $1 \cdot 0$ & 0.8 & 0.8 & $1 \cdot 0$ & \\
\hline \multicolumn{19}{|c|}{ Breakfast cereals } \\
\hline Mean & $8 \cdot 1$ & $6 \cdot 9$ & $5 \cdot 9$ & 4.5 & 4.7 & \multirow[t]{2}{*}{$<0.05$} & $10 \cdot 6$ & $9 \cdot 3$ & $6 \cdot 7$ & $6 \cdot 4$ & 4.4 & \multirow[t]{2}{*}{$<0.001$} & $10 \cdot 2$ & $6 \cdot 8$ & 5.5 & 4.7 & 3.5 & \multirow{2}{*}{$<0.001$} \\
\hline SE & 1.9 & 1.5 & $1 \cdot 2$ & 1.0 & $1 \cdot 1$ & & 1.4 & $1 \cdot 1$ & 0.8 & 0.7 & 0.6 & & $1 \cdot 6$ & $1 \cdot 0$ & 0.8 & 0.7 & 0.5 & \\
\hline \multicolumn{19}{|l|}{ Fruit juice } \\
\hline Mean & 6.5 & $14 \cdot 8$ & 13.8 & 14.4 & $16 \cdot 3$ & \multirow[t]{2}{*}{ NS } & 8.5 & $10 \cdot 2$ & 13.7 & $15 \cdot 9$ & $17 \cdot 3$ & \multirow{2}{*}{$<0.001$} & $6 \cdot 7$ & 9.8 & $10 \cdot 9$ & $12 \cdot 5$ & $10 \cdot 6$ & \multirow[t]{2}{*}{0.05} \\
\hline SE & 2.9 & 3.8 & 3.9 & 3.3 & 3.7 & & 1.9 & 1.7 & 1.9 & 1.9 & $2 \cdot 2$ & & 1.5 & $1 \cdot 6$ & 1.6 & 1.9 & $1 \cdot 7$ & \\
\hline \multicolumn{19}{|c|}{ Soft drinks (non-diet) } \\
\hline Mean & 5.6 & 7.4 & $6 \cdot 1$ & $13 \cdot 0$ & $20 \cdot 8$ & \multirow[t]{2}{*}{$<0.001$} & 11.6 & 13.5 & 14.0 & $18 \cdot 3$ & 22.4 & \multirow[t]{2}{*}{$<0.001$} & $15 \cdot 6$ & 23.7 & 31.2 & 33.0 & $41 \cdot 1$ & \multirow[t]{2}{*}{$<0.001$} \\
\hline SE & 3.0 & 2.5 & $2 \cdot 1$ & 3.0 & $4 \cdot 3$ & & $2 \cdot 1$ & $2 \cdot 0$ & 1.9 & $2 \cdot 1$ & $2 \cdot 4$ & & $2 \cdot 3$ & $2 \cdot 5$ & $2 \cdot 7$ & $2 \cdot 6$ & $2 \cdot 6$ & \\
\hline \multicolumn{19}{|c|}{ Sugar confectionery } \\
\hline Mean & $2 \cdot 2$ & $2 \cdot 0$ & 4.5 & 6.8 & 7.9 & \multirow[t]{2}{*}{$<0.01$} & $2 \cdot 7$ & $5 \cdot 8$ & 7.6 & $9 \cdot 1$ & $10 \cdot 7$ & \multirow[t]{2}{*}{$<0.001$} & 3.8 & 3.5 & $5 \cdot 0$ & 6.4 & $7 \cdot 2$ & $<0.01$ \\
\hline SE & 1.6 & 1.2 & 1.9 & $2 \cdot 2$ & $2 \cdot 3$ & & 0.9 & $1 \cdot 1$ & $1 \cdot 3$ & 1.5 & 1.7 & & $1 \cdot 2$ & 0.9 & $1 \cdot 1$ & 1.3 & 1.4 & \\
\hline Chocolat & & & & & & & & & & & & & & & & & & \\
\hline Mean & 5.4 & 8.9 & 7.8 & 8.2 & $5 \cdot 8$ & NS & 8.8 & 7.5 & $7 \cdot 7$ & $6 \cdot 1$ & 8.4 & NS & 7.6 & $8 \cdot 1$ & 9.5 & $9 \cdot 3$ & 6.9 & NS \\
\hline SE & 2.3 & $2 \cdot 3$ & $2 \cdot 1$ & $2 \cdot 1$ & 1.3 & & 1.8 & $1 \cdot 1$ & 1.4 & 1.0 & 1.4 & & 1.4 & 1.1 & 1.4 & 1.4 & 0.9 & \\
\hline Buns, cal & & & & & & & & & & & & & & & & & & \\
\hline Mean & 4.9 & $5 \cdot 3$ & 6.4 & 4.8 & 4.4 & NS & $9 \cdot 1$ & 9.3 & 8.7 & $9 \cdot 3$ & $6 \cdot 6$ & NS & 4.7 & $7 \cdot 3$ & 4.7 & 4.9 & $4 \cdot 3$ & NS \\
\hline SE & 1.9 & 1.9 & 1.9 & 1.5 & $1 \cdot 2$ & & 1.5 & 1.5 & $1 \cdot 1$ & $1 \cdot 2$ & 0.8 & & 1.0 & $1 \cdot 1$ & 0.9 & 0.8 & 0.6 & \\
\hline Puddings & & & & & & & & & & & & & & & & & & \\
\hline Mean & 3.8 & $4 \cdot 2$ & $2 \cdot 2$ & 4.0 & $2 \cdot 6$ & NS & 2.6 & 3.2 & $2 \cdot 1$ & 1.9 & 1.9 & NS & 1.5 & 1.8 & 1.3 & 1.4 & 1.0 & NS \\
\hline SE & 1.9 & 1.5 & 1.0 & 1.5 & 1.5 & & 0.8 & 0.7 & 0.5 & 0.5 & 0.5 & & 0.5 & 0.6 & 0.3 & 0.4 & 0.3 & \\
\hline Ice crean & & & & & & & & & & & & & & & & & & \\
\hline Mean & 2.8 & 3.5 & 4.6 & 4.1 & 3.6 & NS & $4 \cdot 1$ & 4.4 & 4.5 & 4.5 & 3.4 & NS & 3.4 & 3.4 & 1.9 & $2 \cdot 4$ & 1.5 & $<0.05$ \\
\hline $\mathrm{SE}$ & 1.4 & $1 \cdot 2$ & 1.5 & 1.3 & $1 \cdot 1$ & & 0.9 & 0.9 & 0.9 & 0.7 & 0.6 & & $1 \cdot 1$ & 0.7 & 0.5 & 0.5 & 0.3 & \\
\hline
\end{tabular}

NS, not significant $(P>0.05)$. 
decrease in micronutrient density and an increase in the prevalence of inadequate intakes with higher added sugars intake, but the nutrients showing an association varied by sex and population group. Nutrients of concern were similar to those found in our study: vitamin $\mathrm{A}, \mathrm{Zn}, \mathrm{Mg}, \mathrm{Fe}, \mathrm{Ca}$ and folate. Older children (11-18 years) especially showed potential shortfall for several nutrients (including $\mathrm{Zn}$ and $\mathrm{Fe}$ ).

Some of our concerns regarding diet quality are consistent with those of Marriott et al. ${ }^{(25)}$, who concluded that higher added sugar intakes were associated with higher proportions of individuals with nutrient intakes below the EAR, but 'the overall high calorie intake and the low quality of the U.S. diet remained the predominant issue'. Mean intakes of added sugar in the USA are higher than free sugars/NMES intakes in the UK, despite the fact that added sugars do not include fruit juice. Hence, their finding that nutrient intake was lower with each $5 \%$ increase in added sugar intake above $5-10 \%$ is consistent with our finding that optimal intakes (with respect to micronutrient adequacy) are about $7-14 \%$ free sugars/NMES. The nutrients of concern also differed between the UK and the USA, partly due to different dietary reference values, food composition, dietary habits and assessment methodologies. More participants in the NHANES have vitamin $\mathrm{C}$ intakes below the EAR but this reflects a higher reference intake compared with the UK; conversely, fewer individuals have low $\mathrm{Fe}$ intakes, probably due to fortification or supplementation practices.

Concerns have been raised previously that low sugars intakes may encourage consumption of fat (sugar-fat see-saw). However, we found similar trends for fat, protein and starch $(\% \mathrm{E})$, supporting the suggestion that these associations are partly a mathematical artefact of expressing intakes in percentage terms ${ }^{(27)}$. On the other hand, absolute intakes of protein and fibre $(\mathrm{g} / \mathrm{d})$ also fell with increasing free sugars intake, at least among 11-18-year-olds, consistent with the micronutrient trends suggesting reduced diet quality. This is not necessarily a direct result of sugars consumption, but of food choices, which determine all nutrient intakes. To improve public health, food-based guidelines should focus on discouraging sources of sugars that are low in nutrients or fibre, such as high-sugar soft drinks, confectionery and alcoholic drinks. This is likely to achieve better nutrient intake than limiting all sugar-containing foods.

\section{Strengths and limitations}

Our study extends current knowledge in this area by including analysis of micronutrient density as well as micronutrient intake. This has shown subtle but potentially important differences in dietary quality across the quintiles when controlling for energy. Although we found linear rather than curvilinear associations as previous studies have shown ${ }^{(8,13,15,16)}$, we could not examine these relationships at very low levels of sugar intakes $(<5 \%$ energy) because of insufficient data.

Our analysis was based on nationally representative data, which were both recent and of high quality. The analysis was based on NMES, rather than 'free sugars', but NMES and free sugars can be assumed to be similar for most practical purposes ${ }^{(2)}$. Indeed, at present, there is no alternative data on UK intakes of free sugars.
NMES slightly overestimates free sugars because sugars in dried fruit do not count as free sugars. At an individual level, a small number of children who have high intakes of dried fruit but low intakes of added sugars or fruit juice could be misclassified as high consumers of free sugars in our analysis. However, the impact on micronutrient intake of diets high in free sugars or NMES is likely to be similar. Conclusions with regard to nutrient adequacy depend on the reference values used for comparison. These are of varying certainty, often based on incomplete data or interpolated from studies on other age groups. The 4-d recording period may be insufficient to evaluate intakes of some nutrients that are infrequently consumed (e.g. vitamin A); this should have little effect on mean estimates of intake but would tend to overestimate the proportion of children below the LRNI. Misreporting was addressed in supplementary analyses, but the sugar/nutrient associations were similar when misreporters were excluded. Samples sizes were insufficient to stratify by sex as well as by age group, but the sexes were equally represented in each quintile. Dietary habits may differ between sexes at older ages, and adolescent girls are more likely to be at risk of inadequate micronutrient intakes than boys because of their lower food intake and high requirement for certain nutrients such as $\mathrm{Fe}^{(29)}$. Finally, the usual caveats apply to these cross-sectional data, in that the associations between sugars and micronutrient or EI cannot be assumed to be causal. Low free sugars consumers had the lowest EI, consistent with the policy rationale of using free sugars reduction to drive down EI. However, under-reporting may be a confounder, because exclusion of low energy reporters reduced the observed differences between quintiles. Hence, well-designed intervention studies are needed to evaluate whether lowering free sugars intakes in a free-living population, through such initiatives as the proposed UK tax on sugar-sweetened beverages, leads directly to beneficial and meaningful changes in EI or diet quality, especially among vulnerable groups. Previous studies have identified low compliance in sugar reduction interventions ${ }^{(6)}$.

\section{Conclusions}

Overall, these cross-sectional data showed inverse associations between free sugars intakes and nutrient densities among British children. Intakes of free sugars $>13 \%$ of energy (corresponding to Q3) compared with $<10 \%$ energy (corresponding to Q1) were associated with lower intakes of several nutrients among 4-18-year-olds. Among 11-18-year-olds, there may be nutrients of concern regardless of the level of sugars intake, suggesting that nutrient density requires improvement generally. Poor-quality diets need to be addressed by multiple approaches including changes in dietary habits, reformulation and possibly by fortification or supplementation for some nutrients. On the basis of the present findings, a special focus may be warranted on overconsumption of sugary foods and drinks of low nutrient density.

\section{Acknowledgements}

The NDNS is jointly funded by Public Health England and the Food Standards Agency and made available by the UK Data 
Archive. The data creators, depositors, copyright holder and UK Data Archive bear no responsibility for their further analysis or interpretation.

This work was supported by a research grant from Sugar Nutrition UK.

S. G. designed the study, analysed the data and wrote the article. L. F. contributed to data analysis and literature review. K. N. contributed to literature research and reviewed the article. B. L. contributed to reviewing the article. All the authors approved the final version of the manuscript.

S. G. is Director of Sig-Nurture Ltd, an independent research consultancy to the food industry, government, and not-for-profit and trade organisations. Sig-Nurture Ltd. has received grants and consulting fees in the past 36 months from food, beverage and ingredients manufacturers and trade organisations with an interest in sugar-containing foods and/or sugar replacements. L. F. is an employee of Sig-Nurture Ltd. K. N. is an employee of Sugar Nutrition UK. B. L. is Emerita Professor of Nutrition at Ulster University and has received grants in the past 36 months from Danone and Sugar Nutrition UK.

\section{Supplementary material}

For supplementary material/s referred to in this article, please visit http://dx.doi.org/doi:10.1017/S0007114516003184

\section{References}

1. World Health Organization (2015) Guideline: Sugars Intake for Adults and Children. Geneva: WHO.

2. Scientific Advisory Committee on Nutrition (2015) Carbohydrates and Health Report. London: Department of Health. https://www. gov.uk/government/uploads/system/uploads/attachment_data/ file/445503/SACN_Carbohydrates_and_Health.pdf.

3. US Department of Agriculture (2015) Scientific report of the 2015 Dietary Guidelines Advisory Committee. http://www.health.gov/ dietaryguidelines/2015-scientific-report/PDFs/ (accessed February 2015).

4. Department of Health (1989) Dietary Sugars and Human Disease. Report of the Panel on Dietary Sugars. London: HMSO.

5. Department of Health (1991) Dietary Reference Values for Food Energy and Nutrients for the United Kingdom. Report on Health and Social Subjects, no. 14. London: HMSO.

6. Te Morenga L, Mallard S \& Mann J (2013) Dietary sugars and body weight: systematic review and meta-analyses of randomised controlled trials and cohort studies. BMJ 346, e792.

7. Gibson S (1993) Consumption and sources of sugars in the diets of British schoolchildren: are high-sugar diets nutritionally inferior? J Hum Nutr Diet 6, 355-371.

8. Gibson S (2001) Dietary sugars and micronutrient dilution in normal adults aged 65 years and over. Public Health Nutr $\mathbf{4}$, $1235-1244$.

9. Alexy U, Sichert-Hellert W \& Kersting M (2003) Associations between intake of added sugars and intakes of nutrients and food groups in the diets of German children and adolescents. Br J Nutr 90, 441-447.

10. Kranz S, Smiciklas-Wright H, Siega-Riz AM, et al. (2005) Adverse effect of high added sugar consumption on dietary intake in American preschoolers. J Pediatr 146, 105-111.
11. Gibson SA (2007) Dietary sugars intake and micronutrient adequacy: a systematic review of the evidence. Nutr Res Rev 20, 121-131.

12. Rennie KL \& Livingstone MB (2007) Associations between dietary added sugar intake and micronutrient intake: a systematic review. Br J Nutr 97, 832-841.

13. Gibson S \& Boyd A (2009) Associations between added sugars and micronutrient intakes and status: further analysis of data from the National Diet and Nutrition Survey of Young People aged 4 to 18 years. Br J Nutr 101, 100-107.

14. Forshee RA \& Storey ML (2001) The role of added sugars in the diet quality of children and adolescents. J Am Coll Nutr 20, 32-43.

15. Gibson SA (1997) Do diets high in sugars compromise micronutrient intakes? Micronutrient intakes in the Dietary and Nutritional Survey of British Adults according to dietary concentration of 'added', 'non-milk extrinsic' or 'total' sugars. J Hum Nutr Diet 10, 125-133.

16. Institute of Medicine (2002) Dietary Reference Intakes for Energy, Carbohydrate, Fat, Fibre, Fatty Acids, Cholesterol, Protein and Amino Acids. Washington, DC: Food and Nutrition Board.

17. Public Health England (2015) Why 5\%? https://www. gov.uk/government/publications/sacns-sugars-and-healthrecommendations-why-5 (accessed July 2015).

18. Public Health England (2015) Sugar reduction: the evidence for action. https://www.gov.uk/government/uploads/system/ uploads/attachment_data/file/470179/Sugar_reduction_The_ evidence_for_action.pdf (accessed July 2015).

19. Wittekind A \& Walton J (2014) Worldwide trends in dietary sugars intake. Nutr Res Rev 27, 330-345.

20. Fitt E, Cole D, Ziauddeen N, et al. (2015) DINO (Diet In Nutrients Out) - an integrated dietary assessment system. Public Health Nutr 18, 234-241.

21. NatCen Social Research MRC Human Nutrition Research \& University College London Medical School (2014) National Diet and Nutrition Survey Years 1-4, 2008/09-2011/12, 6th ed. Colchester, Essex: UK Data Archive.

22. Henry CJ (2005) Basal metabolic rate studies in humans: measurement and development of new equations. Public Health Nutr 8, 1133-1152.

23. Scientific Advisory Committee on Nutrition (2011) Dietary Reference Values for Energy. London: TSO. http://www.sacn. gov.uk/

24. Black AE (2000) Critical evaluation of energy intake using the Goldberg cut-off for energy intake:basal metabolic rate. a practical guide to its calculation, use and limitations. Int J Obes Relat Metab Disord 24, 1119-1130.

25. Marriott BP, Olsho L, Hadden L, et al. (2010) Intake of added sugars and selected nutrients in the United States, National Health and Nutrition Examination Survey (NHANES) 2003-2006. Crit Rev Food Sci Nutr 50, 228-258.

26. Louie JC \& Tapsell LC (2015) Intake of total and added sugars and nutrient dilution in Australian children and adolescents. Br J Nutr 114, 1875-1886.

27. Louie JC \& Tapsell LC (2015) Association between intake of total vs added sugar on diet quality: a systematic review. Nutr Rev 73, 837-857.

28. Joyce $\mathrm{T} \&$ Gibney MJ (2008) The impact of added sugar consumption on overall dietary quality in Irish children and teenagers. J Hum Nutr Diet 21, 438-450.

29. Bates BL, Lennox A, Prentice A, et al. (2014) National Diet and Nutrition Survey. Results from Years 1, 2, 3 and 4 (Combined) of the Rolling Programme (2008/2009 to 2011/ 2012). London: PHE publications gateway number 2014051, Public Health England and the Food Standards Agency. 